\title{
Atomic Imaging Across Strain Boundaries in Bilayer Graphene with ADF-STEM and DF-TEM
}

\author{
Robert Hovden ${ }^{1}$, Jonathan Alden ${ }^{1}$, Adam W. Tsen ${ }^{1}$, Pinshane Y. Huang ${ }^{1}$, Lola Brown ${ }^{3}$, Jiwoong Park ${ }^{3,4}$, \\ Paul L. McEuen ${ }^{2,4}$, David A. Muller ${ }^{1,4}$ \\ ${ }^{1}$ School of Applied \& Engineering Physics, Cornell University, Ithaca NY \\ 2 Department of Physics, Cornell University, Ithaca NY \\ ${ }^{3}$ Department of Chemistry and Chemical Biology, Cornell University, Ithaca NY \\ ${ }^{4}$ Kavli Institute at Cornell University, Ithaca NY
}

Bilayer graphene possesses two degenerate lowest energy configurations which are geometrically mirrored-AB and BA stacking[1]. Both stacking geometries have been observed as domains in bilayer systems grown by chemical vapor deposition[2]. Here we examine the structure at the interfaces of stacking domains using atomic-resolution scanning transmission electron microscopy (STEM) and standard dark-field TEM[3]. We find that domain boundaries are formed by a continuous strain between layers with a displacement direction and magnitude quantized by the bilayer energy landscape. The strained interfaces between stacking domains are often microns in length and have been predicted to contain unique electronic properties[4].

Aberration-corrected annular STEM provided direct atomic imaging ( $\sim 1.3-\AA$ probe size) of the strain boundaries between adjacent stacking domains in bilayer graphene. Both tensile and shear strain was experimentally observed in the transition between $\mathrm{AB}$ and BA domains using an annular dark-field (ADF) detector (Fig 1a,d). On either side of the strain boundary, a hexagonal lattice of bright spots represent the ordered $\mathrm{AB}$ and $\mathrm{BA}$ stacking domains. In these ordered regions, the $\mathrm{ADF}$ intensity is enhanced from coherently scattered electrons. Toward the center of the strain boundary the brightness decreases as the image pattern evolves from hexagonal to linear features-horizontal for the shear boundary and vertical for the tensile boundary. The linear patterns arise from the near-overlap of atoms that occurs as the two layers translate across each other vertically and horizontally (Fig. 3c and 3f) [5]. Measured tensile and shear boundary widths provide empirical measurement of the Young's and shear modulus[3]. Quantum multislice simulations show excellent agreement with the data (Fig 1b,e).

The bilayer stacking can be imaged over large fields-of-view using dark-field transmission electron microscopy (DF-TEM)[2]. Apertures placed around diffracted beams are used to form images that reveal stacking domains $\left(1^{\text {st }}\right.$ order Bragg peaks [-1010]) -Figure $2 \mathrm{a}$ - and the boundaries between them $\left(2^{\text {nd }}\right.$ order Bragg peaks [-2110]). Additionally, the [-2110] $2^{\text {nd }}$ order peaks infer the direction of strain across each domain boundary. The color-composite image, Fig. 2b, combines images from three [-2110] diffraction spots colored red, blue, and green, with the relative strain translation annotated by black arrows. Fig. $2 \mathrm{~b}$ shows the core of a topological defect where six domains meet (highlighted by colored arrows). At this point defect, ADF-STEM images reveal a hexagonal lattice characteristic of AAstacked bilayer (Fig 2e) — considerably different from the surrounding AB and BA domains (Fig 2d). The AA-stacked core is very high-energy and manifests from the six $\mathrm{AB}$ and $\mathrm{BA}$ domains surrounding it.

[1] Lebedeva et al., J. Chem. Phys. 134, 104505 (2011)

[2] Brown, et al. Nano Lett. 12, 1609 (2012) 
[3] Alden, et al., Proc. Natl. Acad. Sci. U.S.A. 110, 11256 (2013)

[4] Vaezi, et al., Phys. Rev. X, 3, 021018 (2013)

[5] Lin, et al., Nano Lett., 13, 3262 (2013)

[6] Funded by CCMR NSF MRSEC DMR-1120296, AFOSR FA9550-09-1-0691 and FA9550-10-10410

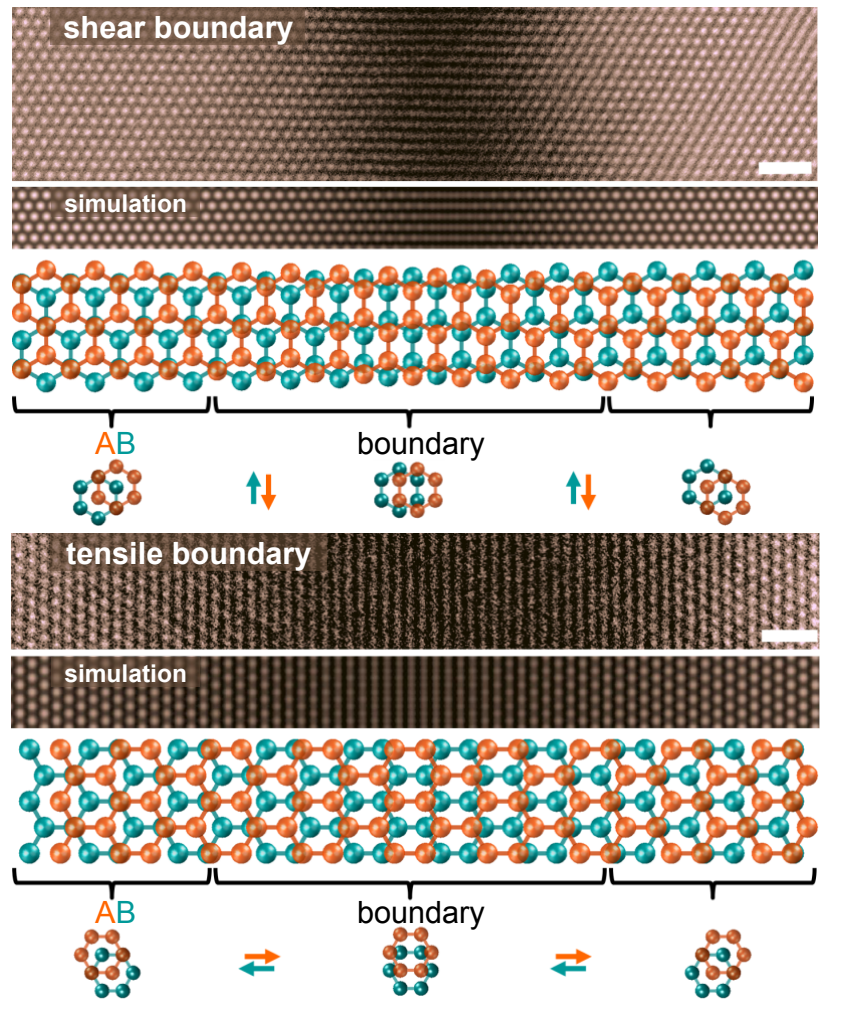

Figure 1 | Atomic-resolution STEM images (A,D) of AB-BA domain boundaries, exhibiting interlayer shear strain and tensile strain, respectively. As one moves across the boundary from left to right, the two sheets translate relative to each other in opposite directions, as indicated by the schematics in $\mathrm{C}$ and $\mathrm{F}$. Each image is an average of four adjacent regions along a boundary. Simulated STEM images of shear and tensile boundaries (B and E) show good agreement with the experimental images. In shear strain (A-C), from left to right, the orange lattice translates downward, whereas the teal lattice translates upward, completing one bondlength interlayer translation (armchairdirection) from $\mathrm{AB}$ to BA. Similarly, in tensile strain (D-F), the orange lattice translates to the right, whereas the teal lattice translates to the left. Figure modified from ref [3].
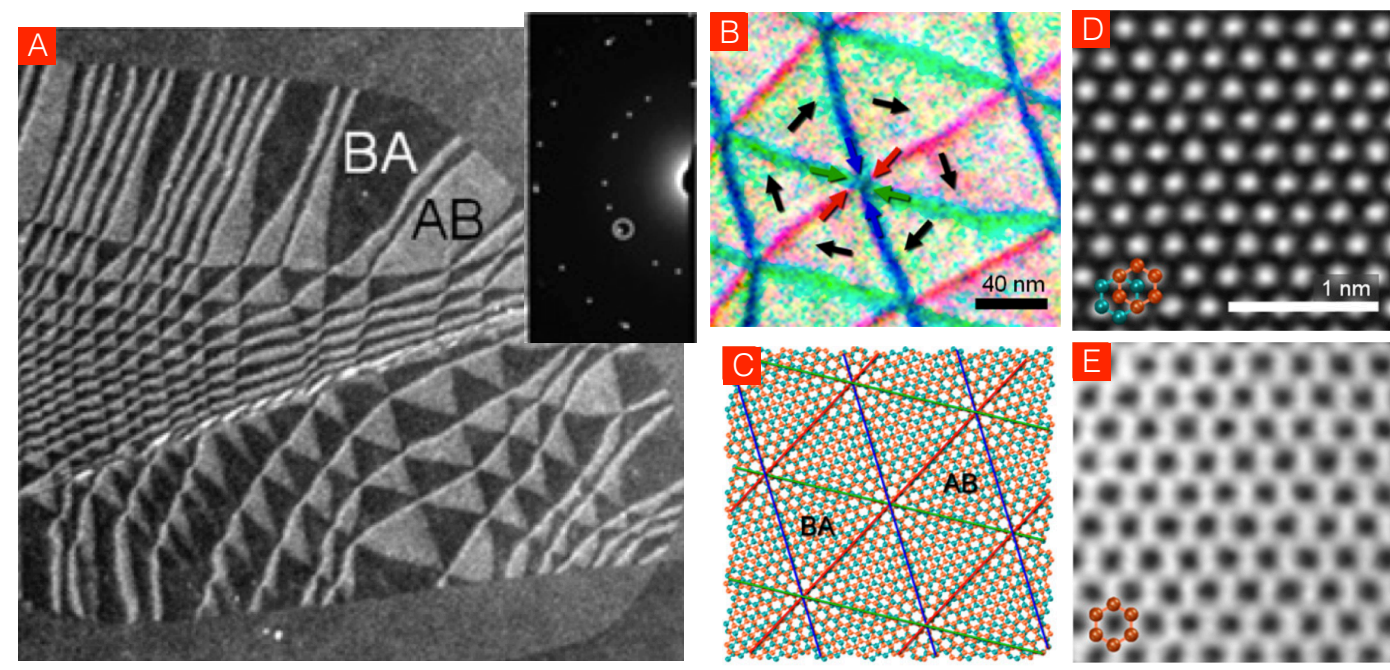

Figure 2 (A) DF-TEM image of bilayer graphene taken from 1st order Bragg spot. (B) An enlarged region of $\mathrm{A}$ taken from $2^{\text {nd }}$ order Bragg spots, showing a defect where six domains meet. Each boundary (red, green, blue) corresponds to an interlayer translation to a neighboring domain (white). (C) Schematic of two rotated graphene sheets, shows a Moiré pattern that is topologically equivalent to the structure in Fig. 2A. Alternating AB- and BAlike regions surround an energetically costly AA stacked core (D). A nearby Bernal-stacked region, for reference $(\mathrm{E})$. C, D have been averaged and low-pass filtered. Figure modified from ref [3]. 